\title{
Detection of SARS-CoV-2 in Saliva by RT-LAMP During a Screening of Workers in Brazil, Including Pre-Symptomatic Carriers
}

\author{
Carlos A. dos Santos, ${ }^{\#, a}$ Kézia G. de Oliveira, ${ }^{\#, b}$ Geovana M. Mendes, ${ }^{\#, b}$ Lívia C. Silva, ${ }^{\#, b}$ \\ Marcio N. de Souza Jr., ,b Paulo Felipe N. Estrela, ${ }^{\#, b}$ Rafael A. Guimarães, ${ }^{c}$ \\ Elisângela P. Silveira-Lacerda ${ }^{a}$ and Gabriela R. M. Duarte ${ }^{\circledR} * b$ \\ ${ }^{a}$ Instituto de Ciências Biológicas, Universidade Federal de Goiás, 74690-900 Goiânia-GO, Brazil \\ ${ }^{b}$ Instituto de Química, Universidade Federal de Goiás, 74690-900 Goiânia-GO, Brazil \\ ${ }^{c}$ Faculdade de Enfermagem, Universidade Federal de Goiás, 74605-080 Goiânia-GO, Brazil
}

\begin{abstract}
The coronavirus pandemic has been causing damage to many nations, as public and private health systems deteriorate by the increasing demand. Some infected patients have culturable severe acute respiratory syndrome coronavirus-2 (SARS-CoV-2) even though not presenting any symptoms, and therefore, are probably able to transmit it. Correctly diagnosing and isolating infected patients is an important step towards preventing new infections. Current diagnostic methods rely mainly on reverse transcription quantitative polymerase chain reaction (RT-qPCR). Methods such as reverse transcription loop-mediated isothermal amplification (RT-LAMP) have risen as viable alternatives, as they are cheaper and require less infrastructure, they have the potential to be applied in low-resource scenarios and even at point-of-care. Here we report a colorimetric RT-LAMP assay capable of detecting SARS-CoV-2 in ribonucleic acid (RNA) from saliva. In some cases, the test was able to detect viral RNA before symptom onset and even in a self-reported asymptomatic carrier. It had a limit of detection of 300 copies per reaction and showed a sensitivity of $80 \%$, a specificity of $100 \%$, a general accuracy of $99.59 \%$, and a Cohen's kappa of 0.887 . The possibility of detecting positive cases even before the clinical manifestation shows great potential and can contribute to controlling the pandemic.
\end{abstract}

Keywords: screening, molecular diagnosis, isothermal amplification, COVID-19 test

\section{Introduction}

In December 2019, a new coronavirus named severe acute respiratory syndrome coronavirus-2 (SARSCoV-2) was detected in Wuhan, Hubei province, China. Since then, the virus has spread rapidly around the world and the World Health Organization (WHO) has declared the disease caused by the new coronavirus 2019 (COVID-19) as a pandemic in March 2020. ${ }^{1}$ The main route of transmission for SARS-CoV-2 is through respiratory droplets carrying infectious virus that might be released into the air when people cough, sneeze, breathe or speak, ${ }^{2}$ therefore, presenting high transmissibility. The high number of SARS-CoV-2 infections is putting pressure on the healthcare system, in addition to leading to major health, social and economic consequences

*e-mail: gabriela_duarte@ufg.br

"The authors contributed equally to this work. for countries. ${ }^{3,4}$ As of 10 May 2021, there have been $157,973,438$ confirmed cases of COVID-19, including $3,288,455$ deaths. $^{5}$

Patients infected with SARS-CoV-2 can develop coronavirus disease (COVID-19). It has a broad clinical spectrum, ranging from a mild disease characterized by symptoms such as fever, cough, sore throat, congestion or runny nose, muscle or body aches, ageusia and/or anosmia, to a severe disease characterized by severe respiratory failure, need for hospitalization, intensive care and invasive mechanical ventilation, which can lead to death. ${ }^{6}$ Also, during the pandemic, asymptomatic cases have been reported and although these patients do not demonstrate clinical manifestation of the disease, recent researches have shown that they are responsible for about 17 to $20 \%$ of new infections. ${ }^{7,8}$ The imposition of non-pharmaceutical interventions (NPIs), such as lockdowns, social distancing, contact tracing, use of face masks and mass testing are key points for controlling COVID-19., ${ }^{3,4}$ 
Current diagnostic tests for SARS-CoV-2 detection rely mainly on reverse transcriptase quantitative polymerase chain reaction (RT-qPCR) as a gold-standard technique for nucleic acid amplification. This technique can detect SARS-CoV-2 with high sensitivity and specificity, and most primer sets can detect at least 100 viral copies. ${ }^{10}$ However, the cost, complexity and facility requirements to use this test on a large scale are major drawbacks for its wide implementation in developing countries. Other nucleic acid amplification methods such as reverse transcription loop-mediated isothermal amplification (RT-LAMP) have been proposed as viable alternatives to RT-qPCR. In addition to being cheaper, there is no need for complex, expensive instrumentation to perform RT-LAMP.

In this study, we evaluated the use of the RT-LAMP assay using ribonucleic acid (RNA) extracted from saliva samples as a tool for screening symptomatic and asymptomatic workers. We obtained data regarding COVID-19 related symptoms through a questionnaire, and we correlated symptom onset to positivity, reporting the efficiency of the test in helping separate positive cases from others.

\section{Experimental}

\section{Biological samples}

Nasopharyngeal swabs of 485 essential workers that, although not in the front line, kept working even during lockdown periods were collected. Participants were also asked to self-collect approximately $1 \mathrm{~mL}$ of saliva. All samples were stored in a cryotube containing $1 \mathrm{~mL} 0.9 \% \mathrm{NaCl}$ solution (LBS Laborasa, São Paulo, Brazil). To inactivate the virus, all samples were incubated at $65{ }^{\circ} \mathrm{C}$ for $1 \mathrm{~h}$ before analysis. Of the total 485 samples tested, 72 samples were from the public transportation company, 121 were from the town's garbage collection company, 78 were from Federal Highway Patrol officers, 174 were from bus drivers working in public transportation and 40 were from employees at the Federal University of Goiás. All workers were interviewed before sample collection and data related to COVID-related symptoms such as fever, dyspnea, sore throat, runny nose, nasal congestion, chest pain, abdominal pain, myalgia, arthralgia, fatigue, headache, loss of smell, loss of taste, diarrhea, nausea, vomiting, conjunctivitis or decreased urine output were collected (Supplementary Information section, Table S1). This study was approved by the Research Ethics Committee of the Federal University of Goiás, protocol number 4.111.485/2020. Furthermore, all workers agreed to participate in the study with a written statement.

\section{RT-LAMP primer designing}

We designed RT-LAMP primers for the detection of SARS-CoV-2 using the LAMP Designer Software, ${ }^{11}$ targeting the $\mathrm{S}$ gene. The six specific primers were synthesized by Integrated DNA Technologies (Coralville, USA). Primer sequences are displayed in Table 1.

\section{Colorimetric detection RT-LAMP for SARS-CoV-2}

RNA from saliva samples for the RT-LAMP test was extracted using the Viral RNA + DNA Preparation Kit (Cellco, São Carlos, Brazil), according to the manufacturer's instructions. RT-LAMP reactions were carried out using the WarmStart Colorimetric LAMP 2X Master Mix (New England Biolabs, Hitchin, UK). The final volume of the reaction was $15 \mu \mathrm{L}$, consisting of $7.5 \mu \mathrm{L}$ of the colorimetric LAMP Master Mix, $1.5 \mu \mathrm{L}$ of a 10X primer mix ( $2 \mu \mathrm{M}$ of each F3 and B3, $16 \mu \mathrm{M}$ of each FIP and BIP, and $8 \mu \mathrm{M}$ of each LF and LB), $4.5 \mu \mathrm{L}$ of nuclease-free water (Sigma-Aldrich, St. Louis, USA) and $1.5 \mu \mathrm{L}$ of RNA sample. Reactions were then heated on a thermoblock (Kasvi, São José dos Pinhais, Brazil) for $30 \mathrm{~min}$ at $68^{\circ} \mathrm{C}$. The results of the colorimetric RT-LAMP test were determined visually by the change of the color pink (negative) to yellow (positive).

Table 1. Primers used in the RT-LAMP reaction

\begin{tabular}{lc}
\hline Primer & Sequence $5{ }^{\prime}-3^{\prime}$ \\
\hline F3 & TGTTAACTGCACAGAAGTCC \\
B3 & TGATGGATTGACTAGCTACA \\
FIP & AGCCTGCACGTGTTTGAAATTTGCTATTCATGCAGATCAACT \\
BIP & ATGAGTGTGACATACCCATTGGTTTTGAGGAGAATTAGTCTGAGTCT \\
LF & ACACGCCAAGTAGGAGTA \\
LB & GCAGGTATATGCGCTAGTTAT \\
\hline
\end{tabular}

F3: forward displacement primer; B3: backward displacement primer; FIP: forward inner primer, BIP: backward inner primer; LF: loop forward primer; LB: loop backward primer; A: adenine; C: cytosine; G: guanine; T: thymine. 


\section{RT-qPCR assay}

To perform RT-qPCR, RNA from swab samples was extracted using the QIAmp Viral RNA Mini Kit (Qiagen, Hilden, Germany), according to the manufacturer's instructions. RT-qPCR was performed using the GoTaq Probe 1-Step RT-qPCR Kit (Promega, Charbonnières-lesBains, France) and 2019-nCoV RUO Kit (Integrated DNA Technologies, Coralville, USA). For each sample, three sets of primers were used targeting the N1, N2 regions of SARS-CoV-2 and RNase P as internal human control, according to the CDC 2019-nCoV Real-Time RT-PCR Diagnostic Panel. ${ }^{12}$ RT-qPCR reactions were carried out in a final volume of $20 \mu \mathrm{L}$ containing $3.1 \mu \mathrm{L}$ of nucleasefree water (Sigma-Aldrich, St. Louis, USA), $1.5 \mu \mathrm{L}$ of the primers and probes, $10 \mu \mathrm{L}$ of GoTaq Probe qPCR Master Mix with dUTP, $0.4 \mu \mathrm{L}$ of Go Script RT Mix for 1-Step RT-qPCR and $5 \mu \mathrm{L}$ of the sample. The amplification was performed in a Step-One Real-Time PCR System (Thermofisher Scientific, Rockford, USA), consisting of one cycle of $15 \mathrm{~min}$ at $45^{\circ} \mathrm{C}$ for reverse transcription, followed by one cycle of $2 \mathrm{~min}$ at $95{ }^{\circ} \mathrm{C}$ for enzyme activation, and then 45 cycles of $2 \mathrm{~s}$ at $95^{\circ} \mathrm{C}$ and $30 \mathrm{~s}$ at $55^{\circ} \mathrm{C}$ for amplification. Samples were considered positive when all the three regions amplified.

\section{Limit of detection of the SARS-CoV-2 RT-LAMP assay}

To assess the limit of detection of the RT-LAMP test, we used serial dilutions of a gBlock synthesized by IDT Technologies (Coralville, USA) containing the target sequence. The test was performed in triplicates. The gBlock is a double-stranded DNA with known molecular size and sequence, obtained by high-fidelity synthesis. The number of copies was quantified using the formula in equation 1 .

$\mathrm{N}=\mathrm{C} \times \mathrm{M} \times\left(1 \times 10^{-15} \mathrm{~mol} \mathrm{fmol}^{-1}\right) \times\left(6,02214086 \times 10^{23} \mathrm{~mol}^{-1}\right)(1)$

where $\mathrm{N}$ is the number of copies in $\mu \mathrm{L}^{-1}, \mathrm{C}$ is the concentration in $n g \mu \mathrm{L}^{-1}$ and $\mathrm{M}$ is the molecular weight in fmol $\mathrm{ng}^{-1}$. Both concentration and molecular weight are provided by the manufacturer. The gBlock was used instead of the RNA standard because it is more stable, simplifying storage. The RT-LAMP-amplified products were determined by visual observation and gel electrophoresis 2\% agarose (GBTSciences, Brasília, Brazil) and $0.5 \%$ Tris-ethylenediaminetetraacetic acid (EDTA)-borate (TEB) buffer (GBTSciences, Brasília, Brazil), revealed with GelRed ${ }^{\circledR}$ (Biotium, Fremont, USA) in an Ultra Lum Omega 10 Molecular Imaging System (Ultra Lum Inc., Claremont, USA).

\section{Statistical analysis}

The sensitivity, specificity and prevalence were calculated using the platform SciStat. ${ }^{13}$ The variables used for statistical analysis are (i) true positive samples (number of samples that are positive by both RT-LAMP and RT-qPCR); (ii) false negative samples (number of samples that were negative by RT-LAMP and positive by RT-qPCR); (iii) false positive samples (number of samples that were positive by RT-LAMP and negative by RT-qPCR) and (iv) true negative samples (number of samples that were negative by both methods). Cohen's Kappa value was calculated using the VassarStats platform. ${ }^{14}$

\section{Results and Discussion}

Even though nasopharyngeal swabs have been proposed as the ideal samples to detect SARS-CoV-2, it is an invasive method and can present some risk to health professionals collecting samples. So, to perform RT-LAMP, we decided to use saliva samples since it would be a less invasive test. Furthermore, some studies have demonstrated the reliability of saliva as a good sample to detect SARS-CoV-2. ${ }^{15-18}$ Untreated saliva samples are not ideal for working directly with the colorimetric RT-LAMP, given the natural and broad range of $\mathrm{pH}$ in which saliva can be collected. Acidic saliva samples would turn the $\mathrm{pH}$ indicator in the reaction yellow, even before the amplification step, resulting in an erroneous interpretation of the results. ${ }^{19}$ Another possibility is to add alkalinizing solutions to the samples, increasing their $\mathrm{pH}$ before adding to the reaction. ${ }^{20}$

The limit of detection of the RT-LAMP test was 300 copies of viral RNA per reaction because it was the minimum number of copies that successfully led to a color change in all replicates, as shown in Figure 1a. The limit of detection found here is similar to that reported in other papers that detected SARS-CoV-2 by colorimetric RT-LAMP. ${ }^{21,22}$

To confirm that the change from pink to yellow was due to specific amplification of the target, electrophoresis in agarose gel was performed (Figure 1b). Gel electrophoresis demonstrated a limit of detection of 200 copies of the viral RNA per reaction. Even though reactions with up to 200 copies presented visible amplification in the agarose gel, it was not vigorous enough to bring the $\mathrm{pH}$ indicator to a turning point. That is because the RT-LAMP colorimetric assay uses phenol red as a detection system, a colorchanging $\mathrm{pH}$ indicator molecule. Phenol red is used to identify the $\mathrm{pH}$ change as a result of successful amplification of the target region. In general, the $\mathrm{pH}$ change begins in the polymerization step, in which the enzyme acting 

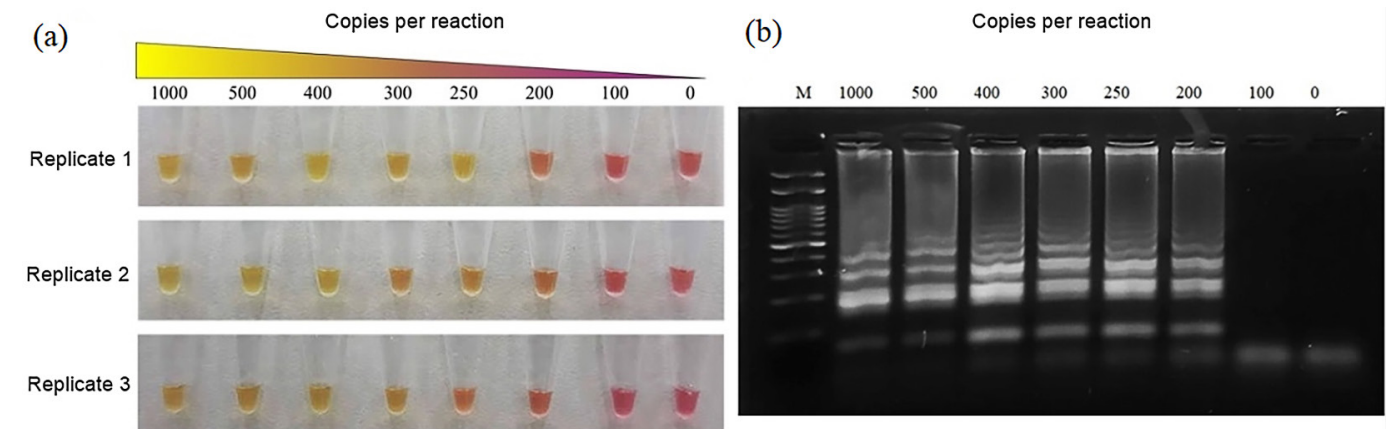

Figure 1. (a) Serial gBlock dilutions to assess the limit of detection of the test. Amplification successfully led to a color change from pink to yellow in the reactions containing at least 300 copies. (b) Agarose gel visualization of the products of the amplification. $\mathrm{M}=100$ base pair molecular marker, $0=$ no template control (nuclease-free water).

in the $5^{\prime} \rightarrow 3^{\prime}$ direction incorporates a deoxynucleotide triphosphate in the target region and promotes the release of two by-products: the pyrophosphate ion, and the hydrogen ion. The interaction between these hydrogen cations $\left(\mathrm{H}^{+}\right)$ and the phenol red indicator yields a gradual color transition from pink to yellow, over the $\mathrm{pH}$ range 7.3 to 6.8. Based on the color change we considered the limit of detection to be 300 copies per reaction. Therefore, despite the occurrence of amplification in reactions containing up to 200 copies (as observed in the agarose gel), the generation of hydronium ion was insufficient to reach the transition interval of the indicator molecule. Even though the limit of detection was lower in the agarose gel when compared to the colorimetric detection, the instrumental ease, low time, no use of mutagenic substances and no manipulation of the reaction after amplification, demonstrate great potential to opt for the colorimetric detection.

A total of 485 clinical samples were analyzed both by RT-qPCR and RT-LAMP. Of the total, 475 (97.9\%) samples were negative and $10(2.1 \%)$ were positive for SARS-CoV-2 by RT-qPCR. Of the 10 samples positive by RT-qPCR, 8 of them were positive by RT-LAMP, as summarized in Table 2 .

Table 2. Comparison between the results of RT-LAMP and the results of RT-qPCR

\begin{tabular}{lccc}
\hline \multirow{2}{*}{ RT-LAMP } & \multicolumn{3}{c}{ RT-qPCR } \\
\cline { 2 - 4 } & Positive & Negative & Total \\
\hline Positive & 8 & 0 & 8 \\
Negative & 2 & 475 & 477 \\
\hline Total & 10 & 475 & 485 \\
\hline
\end{tabular}

RT-LAMP: reverse transcription loop-mediated isothermal amplification; RT-qPCR: reverse transcription quantitative polymerase chain reaction.

Statistical analysis showed a sensitivity of $80 \%$ (95\% confidence interval (CI) from 44.39 to $97.48 \%$ ), a specificity of $100 \%$ (95\% CI from 99.23 to $100.00 \%$ ), a positive predictive value of $100 \%$, a negative predictive value of $99.58 \%$ (95\% CI from 98.57 to $99.88 \%$ ) and overall accuracy of $99.59 \%$. The calculated kappa value was 0.887 (95\% CI from 0.731 to 1.0). All statistical results are summarized in Table 3.

Table 3. Statistical analysis results

\begin{tabular}{lcc}
\hline Statistic & Value & $95 \%$ CI \\
\hline Sensitivity / \% & 80.00 & 44.39 to 97.48 \\
Specificity / \% & 100.00 & 99.23 to 100.00 \\
Positive predictive value / \% & 100.00 & \\
Negative predictive value / \% & 99.58 & 98.57 to 99.88 \\
Accuracy / \% & 99.59 & 98.52 to 99.95 \\
Cohen's Kappa & 0.887 & 0.731 to 1.0 \\
\hline
\end{tabular}

CI: confidence interval.

All samples positive by RT-qPCR with low cycle of quantification $(\mathrm{Cq})(\mathrm{Cq}<30)$ were positive by RT-LAMP, while higher $\mathrm{Cq}$ samples (Cq 32 and $\mathrm{Cq} 34)$ did not show amplification. This is due to the number of copies present in the sample, which are lower than the limit of detection of RT-LAMP, which would be a Cq of about 30 for real samples. Figure 2 shows the clinical samples that were positive by RT-qPCR with their respective $\mathrm{Cq}$ values and the comparison with RT-LAMP visual detection results.

In Figure 3 we show the distribution of all positive samples by RT-qPCR (10) according to the $\mathrm{Cq}$ and the results of the RT-LAMP test. Of the 10 patients that were positive for SARS-CoV-2, 6 patients (patient numbers 80, 171, 233, 273, 295 and 395) reported feeling a medium of 5.17 (95\% CI 4.57 to 5.77) symptoms on the day of collection and were, therefore, symptomatic for the disease. The other 4 did not report any symptoms at the time of collection. These potentially asymptomatic or presymptomatic patients were accompanied through telephone contact, and data regarding COVID-related symptoms was retrieved. Patients 53, 296 and 355 started showing COVID-related symptoms such as headache, cough, fever and muscle pain one day after collection. Patient 355 


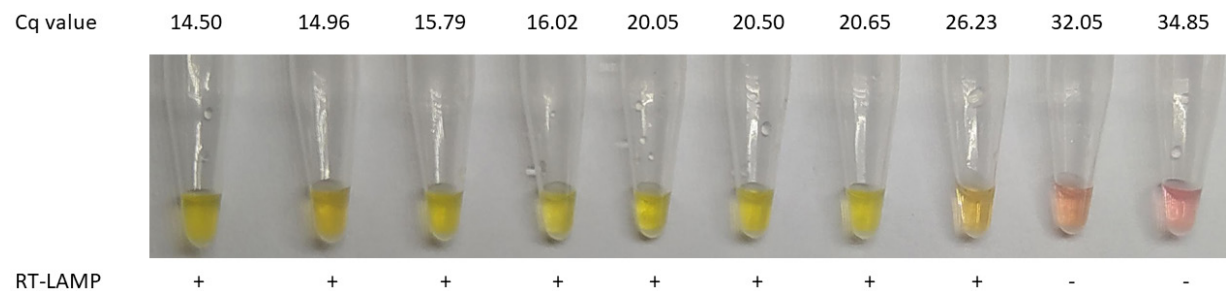

Figure 2. Clinical samples that were positive by RT-qPCR and the comparison with RT-LAMP results. Cq: cycle of quantification; RT-LAMP: reverse transcription loop-mediated isothermal amplification.

reported only feeling a mild headache, and patient number 132 claimed not having felt any symptom at all during the following 14 days after the testing, even though presenting a high viral load. All positive patients were informed of their results within $24 \mathrm{~h}$ from sample collection.

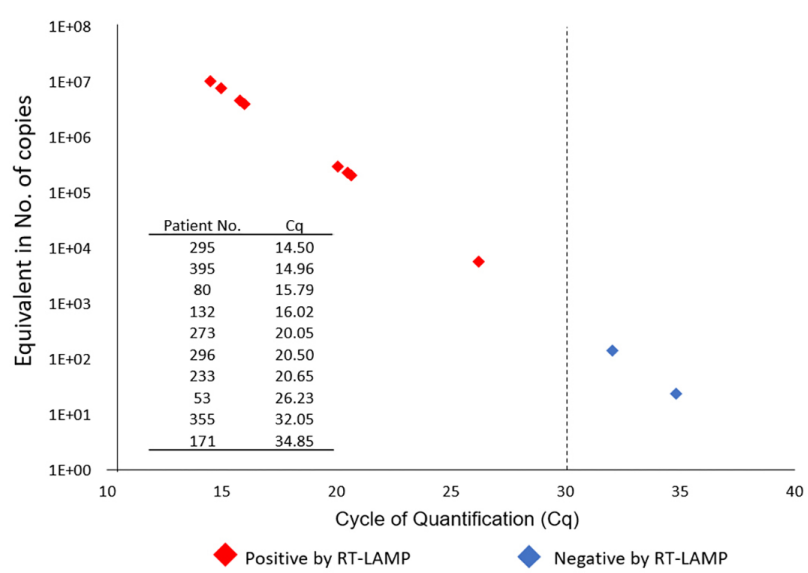

Figure 3. Comparison between RT-LAMP and RT-qPCR. Results of RT-qPCR are median of N1 and N2 Cqs. The number of copies was calculated based on the standard curve. Red diamonds represent samples positive by RT-LAMP and blue diamonds represent samples negative by RT-LAMP. Cq: cycle of quantification; RT-LAMP: reverse transcription loop-mediated isothermal amplification.

Of the 10 patients, 8 had detectable viral loads by both RTLAMP and RT-qPCR on the day of collection, and 2 patients (171 and 355) were negative by RT-LAMP and positive by RT-qPCR. The relatively low number of copies (Cqs 34.85 and 32.05, respectively) can be explained differently based on the patients' symptom onset (Figure 4). Patient 171 had been experiencing symptoms for 14 days when the test was done. It coincides with the shedding duration of the virus. $^{23}$ Therefore, we hypothesize the patient was in the final course of the infection. On the other hand, patient 355 was yet to experience symptoms on the day of collection and therefore was pre-symptomatic. We hypothesize this patient was probably at the beginning of the exponential growth phase of the viral replication. Although the duration of this exponential growth is still not rigorously defined, models of SARS-CoV-2 transmission in ferrets project it to be on the order of a day. ${ }^{24}$ Patients 53 and 296 were detected by RT-LAMP, as they presented Cqs lower than the limit of detection of the test. Due to the higher viral load when compared to patient 355 , we hypothesize these patients were more advanced in the course of the disease.

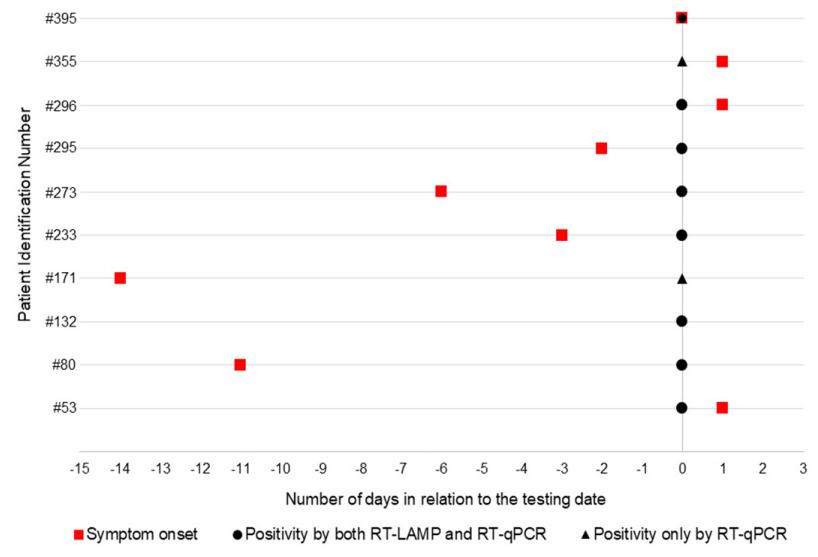

Figure 4. Relation between the number of days from symptom onset and the date of the positive result in a molecular test. Each line represents the course of the disease in one patient. Day 0 is regarded as the testing date. Negative numbers represent the number of days before testing, positive numbers represent the number of days after testing was done. Most patients experienced symptom onset before testing, while three patients (53, 296 and 355) presented a positive result before symptom onset. Patient 395 reported symptom onset on the same day as the testing took place, and patient 132 claimed to never have presented symptoms.

As first pointed out by Larremore et $\mathrm{al}^{25}$ and Mina et al. ${ }^{26}$ it is necessary to rethink our SARS-CoV-2 testing strategy, shifting the focus from how well tests can detect lower quantities of copies to how well tests can detect patients in the infectious period. It is also important to take into account how fast results can be returned, as one of the most effective measures to lower infectivity rates is the isolation of symptomatic, pre-symptomatic and asymptomatic carriers. While developed countries such as the United States peaked at 5.0 tests per thousand people during the pandemic, available data shows that Brazil was hardly able to do 0.48 tests per thousand people. ${ }^{27}$ There is therefore an urgent need to increase testing capacity in countries such as Brazil.

The RT-LAMP assay was able to correctly identify 8 of 10 positive cases, 3 of which did not have symptoms by the time of collection. Even though samples with a $\mathrm{Cq}>30$ did not present positive results in RT-LAMP, it is expected that these patients would have a lower 
probability of transmitting the virus. ${ }^{28-30}$ In this particular study, RT-LAMP results were returned on the same day and positive cases were promptly isolated. We then propose that even not being able to detect these patients with low viral load, the test would still be of help in isolating positive cases, mainly if repeated frequently in a given population. ${ }^{25}$

Our data is limited to its size, and our test fails as it still needs RNA isolation to perform the test. Despite some tests that have shown promising results with a direct swab-to-lamp essay without the need for RNA extraction, ${ }^{31}$ swabs can be difficult and painful to collect, especially for children or people of age. To increase the effectiveness of a testing regimen and the chances of assaying a positive case, SARS-CoV-2 tests would ideally be done repeatedly in an individual on different occasions. Collecting swab samples weekly would have the potential to be a major drawback of testing, as many would probably refuse to have samples collected every couple of days. On the other hand, saliva is much easier to collect and could be collected easily and repeatedly from basically anyone. Therefore, there seems to be a tradeoff between the need for RNA extraction and the simplicity of collection.

To our knowledge, this is the first report that includes the detection of pre-symptomatic and asymptomatic cases of COVID-19 using RT-LAMP and correlates the positivity of the sample with a detailed symptom questionnaire. Although limited to its size, the questionnaire provides enough data to suggest that the test was able to diagnose $2 / 3$ of the pre-symptomatic individuals and one asymptomatic individual. Giving a testing regimen that repeatedly tests individuals, we see the methodology described here as potentially helpful in isolating positive cases, helping in breaking the chain of infection.

\section{Conclusions}

We report an RT-LAMP colorimetric assay that can detect SARS-CoV-2 in RNA from saliva samples from workers at different stages of the infection, including pre-symptomatic carriers, with overall specificity of $100 \%$, sensitivity of $80 \%$ and accuracy of $99.59 \%$. In addition, the method takes less time than the gold standard method (RT-qPCR). Our results show that our colorimetric RT-LAMP assay is suitable for screening large groups, as the great majority of workers were correctly diagnosed based on this simple, fast and low-cost amplification test. Being able to correctly diagnose people carrying transmissible SARS-CoV-2 and quickly isolating them is an important advantage that helps prevent the further spread of the virus, as the contact with others is severely reduced. Due to its low cost and simplicity compared to RT-qPCR,
RT-LAMP could be used in countries that do not have the resources to perform RT-qPCR on a large scale, increasing testing capacity without necessarily increasing costs.

\section{Supplementary Information}

Supplementary information is available free of charge at http://jbcs.sbq.org.br as PDF file.

\section{Acknowledgments}

We are grateful to Nursing School of Universidade Federal de Goiás that kindly collected samples from the population with the team composed of: Rosso, C. F. W., Suzuki, K., Barreto, R. A. S., Guimarães, R. A., Bianchini, A. S. M., Castro, L. I., Reis, A. N., Silva, L. S., Severo, K. M., Alves, A., Guimarães, L. M. S. and Lima, V. L. We are also grateful to Laboratório de Análises Clínicas de Ensino Superior from Universidade Federal de Goiás for the analyzes with the RT-qPCR. This study was supported by the Labor Prosecution Service, CNPq (grant numbers: 402694/2020-1) and FAPEG (grant number: 202010267000273).

\section{Author Contributions}

In this paper, C.A.S., K.G.O., G.M.M., L.C.S., M.N.S.J., P.F.N.E. contributed equally to conceive, design and perform the experiments. C.A.S. designed the primers. C.A.S., K.G.O., G.M.M., L.C.S., P.F.N.E., E.P.S.L. and G.R.M.D. analyzed the data. C.A.S, K.G.O, G.M.M, L.C.S, M.N.C.J., P.F.N.E, R.A.G. wrote the manuscript. All authors reviewed the manuscript.

\section{References}

1. https://www.who.int/director-general/speeches/detail/whodirector-general-s-opening-remarks-at-the-media-briefing-oncovid-19---11-march-2020, accessed in June 2021.

2. Falahi, S.; Kenarkoohi, A.; New Microbes New Infect. 2020, $38,100778$.

3. Flaxman, S.; Mishra, S.; Gandy, A.; Unwin, H. J. T.; Mellan, T. A.; Coupland, H.; Whittaker, C.; Zhu, H.; Berah, T.; Eaton, J. W.; Monod, M.; Perez-Guzman, P. N.; Schmit, N.; Cilloni, L.; Ainslie, K. E. C.; Baguelin, M.; Boonyasiri, A.; Boyd, O.; Cattarino, L.; Cooper, L. V.; Cucunubá, Z.; Cuomo-Dannenburg, G.; Dighe, A.; Djaafara, B.; Dorigatti, I.; van Elsland, S. L.; FitzJohn, R. G.; Gaythorpe, K. A. M.; Geidelberg, L.; Grassly, N. C.; Green, W. D.; Hallett, T.; Hamlet, A.; Hinsley, W.; Jeffrey, B.; Knock, E.; Laydon, D. J.; Nedjati-Gilani, G.; Nouvellet, P.; Parag, K. V.; Siveroni, I.; Thompson, H. A.; Verity, R.; Volz, E.; Walters, C. E.; Wang, H.; Wang, Y.; Watson, O. J.; Winskill, P.; Xi, X.; Walker, P. 
G. T.; Ghani, A. C.; Donnelly, C. A.; Riley, S.; Vollmer, M. A. C.; Ferguson, N. M.; Okell, L. C.; Bhatt, S.; Nature 2020, 584, 257.

4. See, I.; Paul, P.; Slayton, R. B.; Steele, M. K.; Stuckey, M. J.; Duca, L.; Srinivasan, A.; Stone, N.; Jernigan, J. A.; Reddy, S. C.; Clin. Infect. Dis., in press, DOI: 10.1093/cid/ciab110.

5. https://covid19.who.int/, accessed on May 10, 2021.

6. Guan, W.; Ni, Z.; Hu, Y.; Liang, W.; Ou, C.; He, J.; Liu, L.; Shan, H.; Lei, C.; Hui, D. S. C.; Du, B.; Li, L.; Zeng, G.; Yuen, K.-Y.; Chen, R.; Tang, C.; Wang, T.; Chen, P.; Xiang, J.; Li, S.; Wang, J.; Liang, Z.; Peng, Y.; Wei, L.; Liu, Y.; Hu, Y.; Peng, P.; Wang, J.; Liu, J.; Chen, Z.; Li, G.; Zheng, Z.; Qiu, S.; Luo, J.; Ye, C.; Zhu, S.; Zhong, N.; N. Engl. J. Med. 2020, 382, 1708.

7. Buitrago-Garcia, D.; Egli-Gany, D.; Counotte, M. J.; Hossmann, S.; Imeri, H.; Ipekci, A. M.; Salanti, G.; Low, N.; PLoS Med. 2020, 17, e1003346.

8. Byambasuren, O.; Cardona, M.; Bell, K.; Clark, J.; McLaws, M. L.; Glasziou, P.; Off. J. Assoc. Med. Microbiol. Infect. Dis. Can. 2020, 5, 223.

9. Chu, D. K.; Akl, E. A.; Duda, S.; Solo, K.; Yaacoub, S.; Schünemann, H. J.; El-harakeh, A.; Bognanni, A.; Lotfi, T.; Loeb, M.; Hajizadeh, A.; Bak, A.; Izcovich, A.; Cuello-Garcia, C. A.; Chen, C.; Harris, D. J.; Borowiack, E.; Chamseddine, F.; Schünemann, F.; Morgano, G. P.; Muti Schünemann, G. E. U.; Chen, G.; Zhao, H.; Neumann, I.; Chan, J.; Khabsa, J.; Hneiny, L.; Harrison, L.; Smith, M.; Rizk, N.; Giorgi Rossi, P.; AbiHanna, P.; El-khoury, R.; Stalteri, R.; Baldeh, T.; Piggott, T.; Zhang, Y.; Saad, Z.; Khamis, A.; Reinap, M.; Lancet 2020, 395, 1973.

10. Vogels, C. B. F.; Brito, A. F.; Wyllie, A. L.; Fauver, J. R.; Ott, I. M.; Kalinich, C. C.; Petrone, M. E.; Casanovas-Massana, A.; Catherine Muenker, M.; Moore, A. J.; Klein, J.; Lu, P.; LuCulligan, A.; Jiang, X.; Kim, D. J.; Kudo, E.; Mao, T.; Moriyama, M.; Oh, J. E.; Park, A.; Silva, J.; Song, E.; Takahashi, T.; Taura, M.; Tokuyama, M.; Venkataraman, A.; El Weizman, O.; Wong, P.; Yang, Y.; Cheemarla, N. R.; White, E. B.; Lapidus, S.; Earnest, R.; Geng, B.; Vijayakumar, P.; Odio, C.; Fournier, J.; Bermejo, S.; Farhadian, S.; Dela Cruz, C. S.; Iwasaki, A.; Ko, A. I.; Landry, M. L.; Foxman, E. F.; Grubaugh, N. D.; Nat. Microbiol. 2020, 5, 1299.

11. LAMP Designer, v.1.16; Premier Biosoft International, San Francisco, USA, 2011.

12. https://www.fda.gov/media/134922/download, accessed in June 2021.

13. https://www.scistat.com/statisticaltests/diagnostic_test.php, accessed in June 2021.

14. http://vassarstats.net/kappa.html, accessed in July 2021.

15. Yee, R.; Truong, T. T.; Pannaraj, P. S.; Eubanks, N.; Gai, E.; Jumarang, J.; Turner, L.; Peralta, A.; Lee, Y.; Bard, J. D.; J. Clin. Microbiol. 2021, 59, e02686-20.

16. Sohn, Y.; Jeong, S. J.; Chung, W. S.; Hyun, J. H.; Baek, Y. J.; Cho, Y.; Kim, J. H.; Ahn, J. Y.; Choi, J. Y.; Yeom, J.-S.; J. Clin. Med. 2020, 9, 2924.

17. Teo, A. K. J.; Choudhury, Y.; Tan, I. B.; Cher, C. Y.; Chew, S.
H.; Wan, Z. Y.; Cheng, L. T. E.; Oon, L. L. E.; Tan, M. H.; Chan, K. S.; Hsu, L. Y.; Sci. Rep. 2021, 11, 3134.

18. Zhu, J.; Guo, J.; Xu, Y.; Chen, X.; J. Infect. 2020, 81, e48.

19. Uribe-Alvarez, C.; Lam, Q.; Baldwin, D. A.; Chernoff, J.; PLoS One 2021, 16, e0250202.

20. Yang, Q.; Meyerson, N. R.; Clark, S. K.; Paige, C. L.; Fattor, W. T.; Gilchrist, A. R.; Barbachano-Guerrero, A.; Healy, B. G.; Worden-Sapper, E. R.; Wu, S. S.; Muhlrad, D.; Decker, C. J.; Saldi, T. K.; Lasda, E.; Gonzales, P. K.; Fink, M. R.; Tat, K. L.; Hager, C. R.; Davis, J. C.; Ozeroff, C. D.; Brisson, G. R.; McQueen, M. B.; Leinwand, L.; Parker, R.; Sawyer, S. L.; eLife 2021, 10, e65113.

21. Diego, J. G.-B.; Fernández-Soto, P.; Domínguez-Gil, M.; Belhassen-García, M.; Bellido, J. L. M.; Muro, A.; Diagnostics 2021, 11, 438 .

22. Nawattanapaiboon, K.; Pasomsub, E.; Prombun, P.; Wongbunmak, A.; Jenjitwanich, A.; Mahasupachai, P.; Vetcho, P.; Chayrach, C.; Manatjaroenlap, N.; Samphaongern, C.; Watthanachockchai, T.; Leedorkmai, P.; Manopwisedjaroen, S.; Akkarawongsapat, R.; Thitithanyanont, A.; Phanchana, M.; Panbangred, W.; Chauvatcharin, S.; Srikhirin, T.; Analyst 2021, 146, 471.

23. Cevik, M.; Tate, M.; Lloyd, O.; Maraolo, A. E.; Schafers, J.; Ho, A.; Lancet Microbe 2021, 2, e13.

24. Richard, M.; Kok, A.; de Meulder, D.; Bestebroer, T. M.; Lamers, M. M.; Okba, N. M. A.; van Vlissingen, M. F.; Rockx, B.; Haagmans, B. L.; Koopmans, M. P. G.; Fouchier, R. A. M.; Herfst, S.; Nat. Commun. 2020, 11, 3496.

25. Larremore, D. B.; Wilder, B.; Lester, E.; Shehata, S.; Burke, J. M.; Hay, J. A.; Tambe, M.; Mina, M. J.; Parker, R.; Sci. Adv. 2021, 7, eabd5393.

26. Mina, M. J.; Parker, R.; Larremore, D. B.; N. Engl. J. Med. 2020, 383, e120.

27. https://ourworldindata.org/coronavirus, accessed on April 10, 2021.

28. Kim, M.-C.; Cui, C.; Shin, K.-R.; Bae, J.-Y.; Kweon, O.-J.; Lee, M.-K.; Choi, S.-H.; Jung, S.-Y.; Park, M.-S.; Chung, J.-W.; N. Engl. J. Med. 2021, 384, 671.

29. Singanayagam, A.; Patel, M.; Charlett, A.; Bernal, J. L.; Saliba, V.; Ellis, J.; Ladhani, S.; Zambon, M.; Gopal, R.; Eurosurveillance 2020, 25, 2001483.

30. Walsh, K. A.; Spillane, S.; Comber, L.; Cardwell, K.; Harrington, P.; Connell, J.; Teljeur, C.; Broderick, N.; de Gascun, C. F.; Smith, S. M.; Ryan, M.; O’Neill, M.; J. Infect. 2020, $81,847$.

31. Dudley, D. M.; Newman, C. M.; Weiler, A. M.; Ramuta, M. D.; Shortreed, C. G.; Heffron, A. S.; Accola, M. A.; Rehrauer, W. M.; Friedrich, T. C.; O'Connor, D. H.; PLoS One 2020, 15, e0244882.

Submitted: May 12, 2021

Published online: July 7, 2021 